\title{
Computed Tomography-Guided Cryoablation for Adrenal Metastasis Secondary to Non-small-cell Lung Cancer
}

\author{
Jie-Fei Cheng ${ }^{1}$, Wen $\mathrm{Xu}^{2}$ and Pei-Pei Liu ${ }^{3,{ }^{*}}$ \\ ${ }^{1}$ Department of Radiology, The Fourth People's Hospital of Taizhou, Taizhou, 225300 Jiangsu Province, China \\ ${ }^{2}$ Department of Interventional Radiology, Zhejiang Taizhou Hospital, Taizhou, 317000 Zhejiang Province, China \\ ${ }^{3}$ Department of Radiology, Xuzhou Central Hospital, Xuzhou, 221000 Jiangsu Province, China \\ "Corresponding author: Department of Radiology, Xuzhou Central Hospital, Xuzhou, 221000 Jiangsu Province, China. Email: landuo89@163.com
}

Received 2021 January 30; Revised 2021 May 08; Accepted 2021 May 15.

\begin{abstract}
Background: Computed tomography (CT)-guided ablation has been used to treat adrenal metastasis (AM). However, the incidence of AM secondary to non-small-cell lung cancer (NSCLC) has not been investigated.

objectives: To assess the clinical efficacy of CT-guided cryoablation for treating AM secondary to NSCLC.

Patients and Methods: This retrospective study was performed among patients with AM secondary to NSCLC, undergoing CTguided cryoablation in our hospital. The rates of complete ablation, local recurrence, local recurrence-free survival (RFS), systematic RFS, and overall survival (OS) were also analyzed.

Results: Thirty-four consecutive patients with AM secondary to NSCLC (16 cases of squamous cell carcinoma [SCC] and 18 cases of adenocarcinoma) underwent cryoablation in our hospital. The primary complete ablation rates were $93.8 \%$ and $88.9 \%$ in the SCC and adenocarcinoma groups, respectively $(\mathrm{P}=1.000)$. Moderate blood pressure increases were observed in $7 / 34(20.6 \%)$ patients during cryoablation. The local recurrence of AM was observed in five and three patients in the SCC and adenocarcinoma groups, respectively $(\mathrm{P}=0.551)$. The median local RFS was 22 months in all patients, and there was no significant difference in terms of the local RFS between the SCC and adenocarcinoma groups (38 vs. 17 months) $(\mathrm{P}=0.093)$. The median systematic RFS was 28 months in all patients, and the systematic RFS was significantly longer in the SCC group as compared to the adenocarcinoma group (56 vs. 19 months $)(\mathrm{P}=0.001)$. The median OS was 34 months in all patients; it was significantly higher in the SCC group as compared to the adenocarcinoma group (56 vs. 22 months) $(\mathrm{P}=0.009)$.
\end{abstract}

Conclusion: CT-guided cryoablation can be a safe and effective treatment to control AM secondary to NSCLC.

Keywords: Cryoablation, Adrenal Metastasis, Lung Cancer

\section{Background}

Malignant tumors often metastasize to the adrenal glands (1-3), and up to $27 \%$ of patients with known malignant tumors exhibit adrenal metastasis (AM) upon autopsy $(4,5)$. So far, no clinical trial has been conducted to establish the benefits of local AM treatment. However, multiple studies have proposed that surgical resection may improve the survival outcomes in patients with isolated AM (6-10). Laparoscopic adrenalectomy (LA) is commonly used to treat adrenal tumors (6-8). However, it is not an appropriate treatment for patients with certain comorbidities and is associated with prolonged postoperative hospitalization (1). Percutaneous ablation strategies have been used to treat adrenal tumors, with $89-100 \%$ complete ablation rates (11-13). Generally, ablation is less invasive and more cost-effective than LA (14).
Multiple studies have used computed tomography (CT)-guided ablation to treat AM (1-3), achieving local oneyear recurrence-free survival (RFS) rates of $71-82 \%(1,2)$. However, these studies have evaluated AM secondary to multiple primary cancers (e.g., lung, liver, renal, and colorectal cancers), which can potentially cause selection bias in the results (1-3). However, the clinical effectiveness of ablation for AM secondary to a specific malignant tumor has not been evaluated in more detail in previous studies (1-3). In this regard, Hasegawa et al. (1) performed radiofrequency ablation for 35 patients with AM and found that AM from non-small-cell lung cancer (NSCLC) was a significant indicator of poor prognosis. Therefore, it is important to conduct studies to specifically evaluate the treatment of AM secondary to NSCLC. 


\section{Objectives}

This study was designed to assess the efficacy of CTguided cryoablation as a treatment for AM secondary to NSCLC.

\section{Patients and Methods}

The Institutional Review Board approved this retrospective single-center study, and the requirement for written informed consent was waived.

\subsection{Study Design and Eligibility Criteria}

This study enrolled consecutive patients with AM secondary to lung cancer, undergoing CT-guided cryoablation in our hospital between January 2015 and December 2019. The inclusion criteria were as follows: (1) patients who were not eligible for surgery or refused surgical resection; (2) patients who had undergone surgical resection of primary NSCLC; (3) patients with AM $\leq 5 \mathrm{~cm}$ in size; and (4) patients with no extra-adrenal tumors at the time of admission. Patients were excluded if they exhibited adrenal vein invasion or had infections, active bleeding, or significant coagulation disorders.

\subsection{Diagnostic Criteria}

AM secondary to NSCLC was diagnosed based on the patient's history, abdominal CT results, and percutaneous biopsy.

\subsection{Cryoablation Procedure}

Patients were placed in the prone position for access to the posterior anatomic structure, and all procedures were guided by CT scan (Philips, Amsterdam, Netherlands) under local anesthesia. Argon-helium cryoablation (CryoHit, Galil Medical, Israel) was conducted under local anesthesia. Each cryoprobe had a $1.5 \times 3.5 \mathrm{~cm}$ freezing area. Abdominal CT scans were used to locate the tumors in patients and to assess the size of these tumors (Figure 1A). Next, the cryoprobes were distributed according to tumor shape and size (Figure 1B). Since the edge of the freezing area had a temperature of $0^{\circ} \mathrm{C}$, while a temperature below $-40^{\circ} \mathrm{C}$ is necessary to kill the cells, the overall freezing area was approximately $0.5-1 \mathrm{~cm}$ beyond the tumor edge, with a $\leq 1.5 \mathrm{~cm}$ interval between pairs of cryoprobes (3).

Following cryoprobe placement, tumors were subjected to two freeze-thaw cycles (ten minutes of freezing, followed by three minutes of thawing). Freezing was performed through rapid argon gas expansion in the sealed cryoprobes, which reached a minimum tip temperature of $-140^{\circ} \mathrm{C}$ within a few seconds. Thawing was then performed by replacing argon with helium gas. For confirmation of the frozen tissue dimensions, abdominal CT scans were acquired repeatedly following the cryoablation protocols.

Blood pressure increase and hypertensive crisis are common intraoperative complications during ablation for adrenal tumors (1-3). If a patient experiences a moderate blood pressure increase, alpha-blockers should be used, and blood pressure should be monitored until it returns to normal. If the patient experiences a hypertensive crisis, the procedure should be suspended, and $\beta$-blockers, $\alpha$-blockers, hydralazine hydrochloride, and sodium nitroprusside should be used.

\subsection{Postoperative Management}

After cryoablation was completed, the patients were closely monitored for approximately 30 minutes and subsequently returned to the general ward if no discomfort was observed. The patients' vital signs were also monitored for an additional six hours.

\subsection{Follow-up}

Follow-up was routinely conducted from the time of treatment until death or until the last visit during the study. All data were collected in December 2020. Once per month, the patients underwent a standard physical examination and blood collection for laboratory examinations, including blood cell count and adrenal hormone measurements. Besides, postoperative CT scanning was conducted at 1, 3, 6, and 12 months following treatment and every 12 months thereafter.

\subsection{Definitions of Terms}

Complete ablation was defined as the lack of any detectable tumor enhancement upon contrast-enhanced CT evaluation within 3 - 5 days post-treatment (Figure 1C), while residual CT enhancement was an indicator of partial ablation (3). Cryoablation was repeated one week after treatment for cases with a detectable residual enhancement. Local recurrence was defined by the presence of a new tumor enhancement within the ablated region in the first follow-up (3). Local RFS was defined as the time between treatment and the first detection of AM recurrence. Patients who did not experience AM recurrence were considered as event negative. Besides, systematic RFS was defined as the time between treatment and the first detection of extra-adrenal recurrence. Patients who did not experience extra-adrenal recurrence were considered as event 

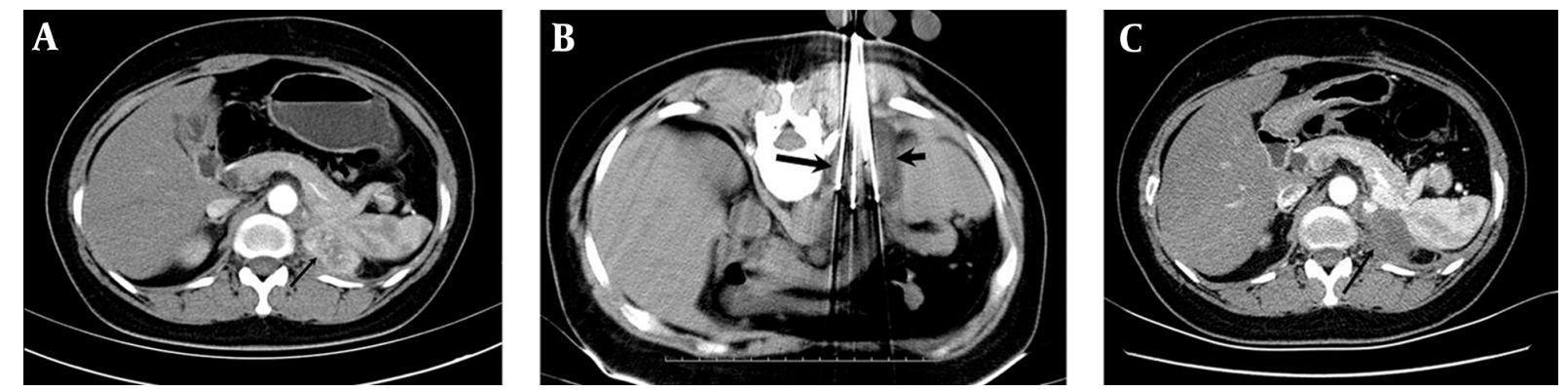

Figure 1. A 59-year-old man with left AM after lung squamous cell carcinoma (SCC) resection. (A) Contrast-enhanced CT images of a left adrenal metastasis (AM) exhibiting significant enhancement (arrow). (B) Cryoablation procedures. The cryoprobes (long arrow) were placed into the tumor, and the frozen area could be seen on the CT image (short arrow). (C) Contrast-enhanced CT scan, indicating no visible tumor enhancement after treatment (arrow).

negative. The overall survival (OS) was also defined as the time between treatment and death or the last follow-up. Patients who were still alive during the follow-up were considered as event negative.

\subsection{Statistical Analysis}

SPSS version 16.0 (SPSS Inc., IL, USA) was used for statistical analysis. Continuous data are presented as mean and compared using $t$-test. Categorical and numerical data were compared via $\chi^{2}$ test and Fisher's exact test. KaplanMeier curves were also used to assess the patients' survival, and a log rank test was used to compare the survival duration. Moreover, a Cox regression analysis was used to assess the association between survival time and one or more predictive factors. Covariates that yielded a P-value $<0.1$ in the univariate analysis remained in the multivariate analysis. The level of statistical significance was set at $\mathrm{P}<0.05$.

\section{Results}

\subsection{Patient Sampling}

Thirty-four consecutive patients with AM secondary to NSCLC, undergoing cryoablation, were analyzed in this study. Each enrolled patient had a single AM. The mean number of used cryoprobes was 2.7 (range: 1 - 4). The primary NSCLCs included squamous cell carcinoma (SCC) $(\mathrm{n}=$ 16) and adenocarcinoma $(n=18)$; there was no other pathological type of primary NSCLC in this study. The baseline data of the patients are presented in Table 1.

The mean and median follow-up of the patients were $28.8 \pm 15.9$ and 22 months, respectively. No patient was lost to the follow-up. The mean follow-up duration was significantly longer in the SCC group as compared to the adenocarcinoma group ( $35.9 \pm 14.9$ vs. $22.6 \pm 14.4$ months $)(\mathrm{P}=$ 0.013). There were six and seven patients in the SCC and adenocarcinoma groups, who underwent chemotherapy after cryoablation, respectively $(\mathrm{P}=0.311)$.

\subsection{Complete Ablation Rate}

The primary complete ablation rates were 93.8\% (15/16) and $88.9 \%$ (16/18) in the SCC and adenocarcinoma groups, respectively $(\mathrm{P}=1.000$ )(Table 2 ). In all patients, the primary complete ablation rate was $91.1 \%$, and the second complete ablation rate was $100 \%$.

\subsection{Procedure-Related Complications}

Moderate blood pressure increases were observed in $7 / 34(20.6 \%)$ patients during cryoablation $(n=5$ in the SCC group and $\mathrm{n}=2$ in the adenocarcinoma group $)(\mathrm{P}=0.306)$. The increase in systolic or diastolic blood pressure was less than $10 \mathrm{mmHg}$ in these seven patients. They were treated with alpha-blockers immediately after the cryoablation procedure was completed and were closely monitored until their blood pressure returned to normal. No patient suffered from a hypertensive crisis, hematoma, hemorrhage, or damage to the surrounding tissues following cryoablation.

\subsection{Local RFS}

The local recurrence of AM was reported in five and three patients in the SCC and adenocarcinoma groups, respectively $(\mathrm{P}=0.551)$. The median time from treatment until local recurrence was 18 months in these eight patients with local recurrence. They were treated with a second cryoablation. The median local RFS was 22 months in all patients, and there was no significant difference in the local RFS between the SCC and adenocarcinoma groups (38 vs. 17 months $)(P=0.093)$. The one-, three-, and five-year local recurrence rates were $0 \%, 20.7 \%$, and $40.5 \%$ in the SCC group, respectively, while the corresponding rates were $11.1 \%, 17.9 \%$, and $17.9 \%$ in the adenocarcinoma group, respectively. 


\begin{tabular}{|c|c|c|c|}
\hline & Squamous cell carcinoma & Adenocarcinoma & P-value \\
\hline Patient number & 16 & 18 & - \\
\hline Age $(y)$ & $59.6 \pm 8.8$ & $57.2 \pm 11.1$ & 0.492 \\
\hline Sex (male/female) & $10 / 6$ & $14 / 4$ & 0.549 \\
\hline Tumor stage in previous surgery & & & 0.405 \\
\hline Stage I & 7 & 4 & \\
\hline Stage II & 6 & 9 & \\
\hline Stage III & 3 & 5 & \\
\hline $\begin{array}{l}\text { Time from previous surgery until } \\
\text { the onset of AM (mo) }\end{array}$ & $20.4 \pm 8.5$ & $19.7 \pm 10.4$ & 0.816 \\
\hline Previous chemotherapy & 6 & 10 & 0.292 \\
\hline Chemotherapy after cryoablation & 6 & 7 & 0.934 \\
\hline Adrenal tumor number & 16 & 18 & - \\
\hline Tumor size $(\mathbf{c m})$ & $2.9 \pm 0.7$ & $3.0 \pm 0.5$ & 0.676 \\
\hline Tumor side & & & 0.681 \\
\hline Left & 10 & 10 & \\
\hline Right & 6 & 8 & \\
\hline
\end{tabular}

Abbreviations: AM, adrenal metastasis.

Table 2. Treatment Characteristics in Patients with Different NSCLC Types

\begin{tabular}{|c|c|c|c|}
\hline & Squamous cell carcinoma & Adenocarcinoma & P-value \\
\hline Patient number & 16 & 18 & - \\
\hline Primary complete ablation & 15 & 16 & 1.000 \\
\hline Secondary complete ablation & 16 & 18 & - \\
\hline Local recurrence & 5 & 3 & 0.551 \\
\hline Extra-adrenal recurrence & 2 & 3 & 1.000 \\
\hline Median local RFS (mo) & 38 & 17 & 0.093 \\
\hline Median systematic RFS (mo) & 56 & 19 & 0.001 \\
\hline Median OS (mo) & 56 & 22 & 0.009 \\
\hline
\end{tabular}

Abbreviations: RFS, recurrence-free survival; OS, overall survival; NSCLC, non-small-cell lung cancer.

\subsection{Systematic RFS}

Extra-adrenal recurrence was observed in two and three patients in the SCC and adenocarcinoma groups, respectively $(\mathrm{P}=1.000)$. The median time from treatment until systematic recurrence was 15 months in these five patients with extra-adrenal recurrence. Four of these patients experienced a recurrence of primary NSCLC, and one patient showed liver metastasis. The median systematic RFS was 28 months for all patients, and the systematic RFS was significantly longer in the SCC group compared to the adenocarcinoma group (56 vs. 19 months) $(\mathrm{P}=0.001)$. The one-, three-, and five-year systematic recurrence rates were $6.2 \%$, $14.8 \%$, and $14.8 \%$ in the SCC group, respectively, while the corresponding rates were $6.7 \%, 42.6 \%$, and $42.6 \%$ in the adenocarcinoma group, respectively.

\subsection{OS Rates}

There were 23 deaths during the study; nine and 14 patients in the SCC and adenocarcinoma groups died due to tumor progression during the follow-up, respectively ( $\mathrm{P}$ $=0.774$ ). The median OS was 34 months in all patients, and the median OS was significantly longer in the SCC group as compared to the adenocarcinoma group (56 vs. 22 months $)(\mathrm{P}=0.009)$. The one-, three-, and five-year mortality rates were $0 \%, 31.2 \%$, and $54.9 \%$ in the SCC group, respectively, while the corresponding rates were $11.1 \%, 83.7 \%$, 
and $83.7 \%$ in the adenocarcinoma group, respectively.

The Cox regression analysis revealed that age (hazard ratio [HR]:1.106; 95\% CI:1.045-1.171; $\mathrm{P}=0.001)$, primary adenocarcinoma (HR: 8.141; 95\% CI: 2.461 - 26.928; P = 0.001), and primary stage III NSCLC (HR: 7.560; 95\% CI: 2.006 28.493; $\mathrm{P}=0.003$ ) were independently predictive of poor OS (Table 3). Surprisingly, chemotherapy following cryoablation was not associated with OS (HR: 2.152; 95\% CI: 0.888 5.213; $\mathrm{P}=0.090)$.

\section{Discussion}

Commonly, AMs are treated via CT-guided radiofrequency ablation and microwave ablation $(1,2,13)$. More recently, cryoablation approaches have been applied to treat AM and other malignant tumors, since they cause less pain, allow for visualization of the treatment zone, and are beneficial for better healing $(3,12)$. Our findings suggested that CT-guided cryoablation can be a safe and effective treatment to control AM secondary to NSCLC. The success rates of primary and secondary ablations in the present cohort were $91.1 \%$ and $100 \%$, respectively. These high success rates might be attributed to the mean AM size in this study (3 $\mathrm{cm})$.

In previous studies regarding CT-guided radiofrequency, microwave ablation, and cryoablation for AM patients, the mean AM size ranged from 3 to $3.7 \mathrm{~cm}$ (1-3). The success rates of primary and secondary ablations in this study are comparable to those reported in other studies of CT-guided radiofrequency, microwave ablation, and cryoablation for AM patients (1-3). Besides, there was no significant difference in the primary ablation rates between patients with SCC and adenocarcinoma $(\mathrm{P}=1.000)$. This finding indicated that the success rate of cryoablation was not influenced by the pathological type of primary NSCLC.

The local recurrence rate was reported in $7 / 34$ (20.5\%) patients in the present cohort, which is comparable to the rate reported in a previous study of microwave/radiofrequency ablation for AM secondary to NSCLC (22\%) (13). Similarly, other studies reported a $20 \%$ local recurrence rate for AM secondary to liver cancer (15). Moreover, we found that the median time until local progression was 18 months, which is within the range reported in studies using microwave ablation for 24 months and radiofrequency ablation for 8.6 months $(1,16)$. Besides, the local recurrence rate $(\mathrm{P}=0.551)$ and local RFS $(\mathrm{P}=0.931)$ were not significantly different between patients with primary SCC and adenocarcinoma in this study. Overall, these results demonstrated that the local recurrence rate and time were not influenced by the ablation method or primary cancer type. The local control rate reached 79.5\% in the present study, which is very close to that reported in patients undergoing adrenalectomy $(77-83 \%)(3,4)$.

Regarding regional recurrence, 5/34 (14.7\%) patients experienced extra-adrenal recurrence. The five-year systematic RFS rates were very low in both groups of patients with primary SCC and adenocarcinoma (11.9\% and 0\%, respectively). Since cryoablation is a local treatment, it has limited effects on distant recurrence. Radiological examination is critical for identifying extra-adrenal recurrence and ensuring that proper treatment is administered on time.

A previous study on CT-guided AM ablation showed that the OS of patients with AM secondary to NSCLC was significantly lower than that of patients with AM secondary to other cancer types (1). In this study, we found that the median OS was 34 months in all patients, which is longer than 18.9 months reported in a previous study (1). This discrepancy might be attributed to different patient inclusion criteria, as all of our patients had undergone surgical resection of primary NSCLC.

We also found that the median OS was significantly longer in the SCC group as compared to the adenocarcinoma group (56 vs. 22 months) $(\mathrm{P}=0.009)$. The Cox regression analysis also revealed that primary SCC was a predictor of longer OS. It was also shown that the NSCLC subtype is an important determinant of metastasis and patient survival. Many studies have reported that lung adenocarcinoma is more prone to lymph node metastasis than SCC (17, 18). This may explain why patients with primary SCC in our study had a longer OS as compared to patients with adenocarcinoma. Age and stage III primary NSCLCs were both independent predictors of OS in our cohort. Age was likely to be an adverse prognostic factor, partly because older patients were often immunocompromised; therefore, tumors in older patients progress faster than younger patients (1).

Chemotherapy is a common procedure to treat NSCLC. However, in our multivariate Cox regression analysis, no significant association was found between chemotherapy and patient survival $(\mathrm{P}=0.090)$. This may be attributed to the limited sample size of this study. Further studies on a larger cohort of patients are warranted to determine whether chemotherapy exerts beneficial effects on patient survival. The ablation of adrenal tumors has been found to be associated with the incidence of a hypertensive crisis $(2,11)$. It is known that a thermal ablation causes significant temperature increases in the target tissues and is associated with higher hypertensive crisis rates as compared to other forms of ablation (3). Several moderate hyperten- 


\begin{tabular}{|c|c|c|c|c|c|c|}
\hline \multirow{2}{*}{ Variables } & \multicolumn{3}{|c|}{ Univariate analysis } & \multicolumn{3}{|c|}{ Multivariate analysis } \\
\hline & Hazard ratio & $\mathbf{9 5} \% \mathrm{CI}$ & P-value & Hazard ratio & 95\% CI & P-value \\
\hline Age & 1.048 & $1.001-1.097$ & 0.047 & 1.106 & $1.045-1.171$ & 0.001 \\
\hline Gender & 0.694 & $0.234-1.796$ & 0.405 & & & \\
\hline Tumor size & 1.655 & $0.810-3.381$ & 0.167 & & & \\
\hline \multicolumn{7}{|l|}{ Tumor side } \\
\hline Right & 1 & & & & & \\
\hline Left & 0.557 & $0.227-1.368$ & 0.202 & & & \\
\hline \multicolumn{7}{|l|}{ Lung cancer } \\
\hline Squamous cell carcinoma & 1 & & & 1 & & \\
\hline Adenocarcinoma & 3.003 & $1.251-7.209$ & 0.014 & 8.141 & $2.461-26.928$ & 0.001 \\
\hline \multicolumn{7}{|l|}{ Tumor stage in previous surgery } \\
\hline Stage I & 1 & & & 1 & & \\
\hline Stage II & 1.652 & $0.587-4.645$ & 0.341 & 1.666 & $0.577-4.809$ & 0.345 \\
\hline Stage III & 3.740 & $1.177-11.887$ & 0.025 & 7.560 & $2.006-28.493$ & 0.003 \\
\hline \multicolumn{7}{|l|}{$\begin{array}{l}\text { Chemotherapy before } \\
\text { cryoablation }\end{array}$} \\
\hline \multicolumn{7}{|l|}{ No } \\
\hline Yes & 1.828 & $0.776-4.304$ & 0.167 & & & \\
\hline \multicolumn{7}{|l|}{$\begin{array}{l}\text { Chemotherapy after } \\
\text { cryoablation }\end{array}$} \\
\hline No & 1 & & & & & \\
\hline Yes & 2.370 & $0.993-5.656$ & 0.052 & 2.152 & $0.888-5.213$ & 0.090 \\
\hline
\end{tabular}

Abbreviation: CI, confidence interval.

sion cases were identified and provided with timely blood pressure control medications. However, no case of a hypertensive crisis was found in the present cohort; therefore, timely blood pressure control after cryoablation may decrease the incidence of a hypertensive crisis (3).

There are several limitations to this study. Firstly, this was a retrospective analysis and is therefore, subjected to selection bias. Secondly, this was a single-center study with a small sample size; therefore, caution must be taken when generalizing the results to a larger patient population or over longer follow-up periods. Thirdly, this study did not include a comparison group of patients with some other forms of ablation; therefore, further multi-center randomized controlled trials are crucial to validate and expand these findings. Fourthly, only some of our patients received postoperative chemotherapy, based on their clinical status. Some of the patients did not receive chemotherapy because of their poor economic status, while some patients refused to receive chemotherapy after cryoablation; this definitely increased the risk of bias.
In conclusion, CT-guided cryoablation can be a safe and effective treatment for AM secondary to NSCLC; patients with AM secondary to SCC may benefit the most from this treatment.

\section{Footnotes}

Authors' Contributions: Study concept and design: P.P.L.; Analysis and interpretation of data: J.F.C and W.X.; Drafting of the manuscript: J.F.C.; Critical revision of the manuscript for important intellectual content: P.P.L.; and Statistical analysis: W.X.

Conflict of Interests: The authors declare no conflict of interest.

Ethical Approval: This study was approved by the Institutional Review Board of the Fourth People's Hospital of Taizhou (TZSY-202012008).

Funding/Support: This study was supported by the Program of 2019 Xuzhou Clinical Technique Research (2109GG002). 
Informed Consent: The requirement for written informed consent was waived because this is a retrospective study.

\section{References}

1. Hasegawa T, Yamakado K, Nakatsuka A, Uraki J, Yamanaka T, Fujimori $\mathrm{M}$, et al. Unresectable adrenal metastases: Clinical outcomes of radiofrequency ablation. Radiology. 2015;277(2):584-93. doi: 10.1148/radiol.2015142029. [PubMed: 25997031].

2. Frenk NE, Daye D, Tuncali K, Arellano RS, Shyn PB, Silverman SG, et al. Local control and survival after image-guided percutaneous ablation of adrenal metastases. J Vasc Interv Radiol. 2018;29(2):276-84. doi: 10.1016/j.jvir.2017.07.026. [PubMed: 28927661].

3. Zhang W, Sun LJ, Xu J, Fu YF, Zhuang ZX. Computed tomographyguided cryoablation for adrenal metastases: local control and survival. Medicine (Baltimore). 2018;97(51). e13885. doi: 10.1097/MD.0000000000013885. [PubMed: 30572560]. [PubMed Central: PMC6320079].

4. Strong VE, D'Angelica M, Tang L, Prete F, Gonen M, Coit D, et al. Laparoscopic adrenalectomy for isolated adrenal metastasis. Ann Surg Oncol. 2007;14(12):3392-400. doi: 10.1245/s10434-007-9520-7. [PubMed: 17665267].

5. Abrams HL, Spiro R, Goldstein N. Metastases in carcinoma; analysis of 1000 autopsied cases. Cancer. 1950;3(1):74-85. doi: 10.1002/1097-0142(1950)3:1<74::aid-cncr2820030111>3.0.co;2-7. [PubMed: 15405683].

6. Gunjur A, Duong C, Ball D, Siva S. Surgical and ablative therapies for the management of adrenal 'oligometastases' - A systematic review. Cancer Treat Rev. 2014;40(7):838-46. doi: 10.1016/j.ctrv.2014.04.001. [PubMed: 24791623].

7. Muth A, Persson F, Jansson S, Johanson V, Ahlman H, Wangberg B. Prognostic factors for survival after surgery for adrenal metastasis. Eur J Surg Oncol. 2010;36(7):699-704. doi: 10.1016/j.ejso.2010.04.002. [PubMed: 20452170].

8. Howell GM, Carty SE, Armstrong MJ, Stang MT, McCoy KL, Bartlett DL, et al. Outcome and prognostic factors after adrenalectomy for patients with distant adrenal metastasis. Ann Surg Oncol. 2013;20(11):3491-6. doi: 10.1245/s10434-013-3050-2. [PubMed: 23793361]. [PubMed Central: PMC4879835].
9. Vazquez BJ, Richards ML, Lohse CM, Thompson GB, Farley DR, Grant $\mathrm{CS}$, et al. Adrenalectomy improves outcomes of selected patients with metastatic carcinoma. World J Surg. 2012;36(6):1400-5. doi: 10.1007/s00268-012-1506-3. [PubMed: 22411083].

10. Moreno P, de la Quintana Basarrate A, Musholt TJ, Paunovic I, Puccini $M$, Vidal O, et al. Adrenalectomy for solid tumor metastases: results of a multicenter European study. Surgery. 2013;154(6):1215-22. discussion 1222-3. doi:10.1016/j.surg.2013.06.021. [PubMed: 24238044].

11. Welch BT, Callstrom MR, Carpenter PC, Wass CT, Welch TL, Boorjian SA, et al. A single-institution experience in image-guided thermal ablation of adrenal gland metastases. JVasc Interv Radiol. 2014;25(4):593-8. doi: 10.1016/j.jvir.2013.12.013. [PubMed: 24507995].

12. Fu YF, Cao C, Shi YB, Zhang W, Huang YY. Computed tomographyguided cryoablation for functional adrenal aldosteronoma. Minim Invasive Ther Allied Technol. 2021;30(3):169-73. doi: 10.1080/13645706.2019.1708754. [PubMed: 31889464].

13. Botsa EI, Thanou IL, Papatheodoropoulou AT, Thanos LI. Thermal ablation in the management of adrenal metastasis originating from nonsmall cell lung cancer: A 5-year single-center experience. Chin Med J (Engl). 2017;130(17):2027-32. doi:10.4103/0366-6999.210496. [PubMed: 28707652]. [PubMed Central: PMC5586169].

14. Chen J, Wu J, Zhu R, Lu L, Ma XJ. Ablation versus laparoscopic adrenalectomy for the treatment of aldosteroneproducing adenoma: a meta-analysis. Abdom Radiol (NY). 2021;46(6):2795-804. doi: 10.1007/s00261-020-02887-8. [PubMed: 33386911].

15. Huang J, Xie X, Lin J, Wang W, Zhang X, Liu M, et al. Percutaneous radiofrequency ablation of adrenal metastases from hepatocellular carcinoma: a single-center experience. Cancer Imaging. 2019;19(1):44. doi: 10.1186/s40644-019-0231-7. [PubMed: 31242934]. [PubMed Central: PMC6595611].

16. Ren C, Liang P, Yu XL, Cheng ZG, Han ZY, Yu J. Percutaneous microwave ablation of adrenal tumours under ultrasound guidance in $33 \mathrm{pa}-$ tients with 35 tumours: A single-centre experience. Int J Hyperthermia. 2016;32(5):517-23. doi: 10.3109/02656736.2016.1164905. [PubMed: 27145838].

17. Bao F, Yuan P, Yuan X, Lv X, Wang Z, Hu J. Predictive risk factors for lymph node metastasis in patients with small size non-small cell lung cancer. J Thorac Dis. 2014;6(12):1697-703. doi: 10.3978/j.issn.20721439.2014.11.05. [PubMed: 25589962]. [PubMed Central: PMC4283338].

18. Cho S, Song IH, Yang HC, Kim K, Jheon S. Predictive factors for node metastasis in patients with clinical stage I nonsmall cell lung cancer. Ann Thorac Surg. 2013;96(1):239-45. doi: 10.1016/j.athoracsur.2013.03.050. [PubMed: 23673071]. 